\title{
GeRminaÇÃo dAS SEMEnTES de Conyza canadensis e Conyza bonariensis EM FUNÇÃO DA QUALIDADE DE LUZ
}

\author{
Germination of Conyza canadensis and Conyza bonariensis Seeds as a Function of Light \\ Quality
}

\begin{abstract}
YAMASHITA, O.M. ${ }^{2}$, GUIMARÃES, S.C. ${ }^{3}$ e CAVENAGHI, A.L. ${ }^{4}$
RESUMO - Conyza canadensis e $C$. bonariensis são espécies invasoras cujo relato tem sido cada vez mais frequente, dada sua capacidade de desenvolvimento em áreas com palhada, sob sistema de semeadura direta (SSD). A luz é necessária para a germinação de diversas espécies de plantas daninhas, sendo considerado um fator de superação da dormência das sementes. A germinação de sementes sob SSD sofre o efeito da filtragem de comprimentos de onda pela palhada, que podem influenciar diretamente esse processo. O presente trabalho teve por objetivo estudar o efeito de diferentes filtros de luz sobre a germinação de sementes de $C$. canadensis e $C$. bonariensis. Foi realizado um experimento em que as sementes das espécies foram colocadas para germinar sobre papel mata-borrão umedecido com água, dentro de caixas gerbox transparentes envolvidas com filtros de luz azul, verde, vermelho, vermelho-distante, transparente e também em caixas de gerbox preta (ausência de luz). Foi avaliada a germinação aos cinco e 10 dias após a semeadura e no final desse período por mais 10 dias, com todos os tratamentos recebendo luz branca. As sementes de ambas as espécies sofrem significativamente o efeito da filtragem de comprimentos de onda, exceto o filtro vermelho, que proporcionou germinação superior à dos demais filtros, sendo inferior apenas ao transparente.
\end{abstract}

Palavras-chave: buva, voadeira, filtro de luz, luz vermelha

\begin{abstract}
The weed species Conyza canadensis and $\mathbf{C}$. bonariensis have been increasingly reported, given their capacity to develop under the no-tillage system (NTS). Light is necessary for germination of several weeds, and is considered a factor of seed dormancy break. Seed germination in NTS is affected by light wave length filtering by straw, which can directly influence that process. The aim of this work was to determine the effect of different light filters on seed germination of C. canadensis and $\mathbf{C}$. bonariensis. An experiment was carried out in which the seeds of these species were placed to germinate on blotting paper moistened with distilled water, inside transparent gerboxes involved with blue, green, red, light red and transparent light filters, as well as black gerboxes (light absence). Seed germination was evaluated at 5 and 10 days after sowing and at the end of this period for 10 more days, with all the treatments being under the condition of white light (filter absence). The seeds of both species were significantly affected by wave length filtering, except for the red filter which provided superior germination, inferior only to transparent filter.
\end{abstract}

Keywords: horseweed, fleabane, light filter, red light.

Recebido para publicação em 26.7.2010 e aprovado em 16.3.2011.

2 Professor, Dr., Dep. de Agronomia, Universidade do Estado de Mato Grosso, Campus de Alta Floresta, Av. Perimetral Rogério Silva s/n, Jd. Flamboyant, 78580-000 Alta Floresta-MT, <yama@unemat.br>; ${ }^{3}$ Professor, Dr., Faculdade de Agronomia e Medicina Veterinária, Universidade Federal de Mato Grosso, Av. Fernando Corrêa da Costa s/n. Coxipó. 78060-900 Cuiabá-MT; ${ }^{4}$ Professor, Dr., Dep. de Agronomia - Univag, Av. Dom Orlando Chaves, 2655, Cristo Rei, 78118-000 Várzea Grande-MT.

Planta Daninha, Viçosa-MG, v. 29, n. 4, p. 737-743, 2011 


\section{INTRODUÇÃO}

A germinação é uma sequência ordenada de atividades metabólicas divididas em fases, que resulta na formação de uma plântula (Bewley \& Black, 1994).

A reduzida porcentagem de germinação ou emergência pode ser uma consequência de problemas como dormência das sementes, baixo vigor ou devido a fatores ambientais, como temperatura, luz e dificuldades de embebição (Menezes et al., 2004).

A luz é necessária para a germinação de algumas espécies, sendo considerada por alguns autores como um fator de superação da dormência das sementes (Bewley \& Black, 1994; Baskin \& Baskin, 1998).

O efeito da luz na germinação das sementes, regulado pelo fitocromo (Takaki, 2001), tem sido estudado em diversas espécies, incluindo interações com temperatura, nitrato e giberelina (Toledo et al., 1993; Casal \& Sánchez, 1998; Takaki, 2001; Lopes et al., 2005). Aparentemente, o fitocromo está associado ao funcionamento das membranas biológicas, regulando sua permeabilidade e controlando o fluxo de inúmeras substâncias dentro das células e entre elas, permitindo ou não a resposta fotomorfogenética (Casal \& Sánchez, 1998).

A sensibilidade da semente ao efeito da luz varia de acordo com a qualidade, a intensidade luminosa e o tempo de irradiação, bem como com o período e a temperatura de embebição (Bewley \& Black, 1994; Baskin \& Baskin, 1998).

A variação da qualidade da luz acontece pela absorção diferencial dos comprimentos de onda do espectro da luz que chegam até as plantas (Casal \& Sánchez, 1998; Merotto Jr. et al., 2002). A energia utilizada no processo fotossintético origina-se dos comprimentos de onda da região do visivel (aproximadamente 400 a $700 \mathrm{~nm}$ ), que corresponde à faixa da luz azul até a vermelha. A radiação com comprimento de onda na faixa da luz vermelha distante (730 a $740 \mathrm{~nm}$ ) é pouco absorvida pelas plantas, sendo, assim, dissipada na forma de reflexão (Takaki, 2001).

A maioria dos solos atenua a luz, impedindo sua penetração em grandes profundidades
(Toledo et al., 1993). Além de a luz penetrar apenas nos primeiros milimetros da superficie do solo, a cobertura vegetal altera a qualidade da luz, agindo como um filtro (Ballaré \& Casal, 2000).

Sementes de plantas daninhas podem persistir no solo por muitos anos e germinar uma vez que a dormência seja quebrada e as condições sejam favoráveis. A luz tem sido relatada como um importante fator para a germinação de sementes de muitas plantas daninhas (Klein \& Felippe, 1991; Toledo et al., 1993; Chachalis \& Reddy, 2000; Koger et al., 2004; Nandula et al., 2006; Ikeda et al., 2008; Yamashita et al., 2008).

C. canadensis e $C$. bonariensis são duas espécies da família Asteraceae que atualmente têm se destacado pelo seu caráter negativo, como importantes plantas daninhas que infestam áreas de cultivo anual e perene, além de pastagens e margens de estradas (Thebaud \& Abbott, 1995; Kissmann \& Groth, 1999). Ambas as espécies são ruderais de sucessão primária, que se estabelecem em áreas perturbadas (Tremmel \& Peterson, 1983). Regiões de tradição agrícola, como o Rio Grande do Sul, têm relatado problemas de controle e reduções na produtividade em cultivo de soja pela presença dessas plantas. $\mathrm{O}$ conhecimento da biologia germinativa dessas espécies pode permitir a adoção de práticas corretas de manejo, sendo uma alternativa ao seu controle químico.

O presente trabalho teve como objetivo avaliar o efeito da qualidade de luz na germinação de sementes de C. canadensis e C. bonariensis.

\section{MATERIAL E MÉTODOS}

O experimento foi desenvolvido entre os meses de janeiro e fevereiro de 2008 em condições de laboratório.

As sementes foram coletadas em outubro de 2007, de plantas espontâneas em áreas de cultivo na região de Alta Floresta-MT. Os capitulos foram colhidos manualmente, quando as sementes estavam prontas para dispersão pelo vento, ou seja, quando agitadas, ocorria o seu desprendimento da inflorescência. Após a colheita, foram deixadas para 
secar à sombra. Foi realizada uma seleção visual, descartando-se as sementes com evidência de danos ou mal formadas. As sementes selecionadas foram embaladas em sacos de papel kraft e armazenadas em câmara refrigerada $\left(12,0 \pm 0,5^{\circ} \mathrm{C}\right.$ e $\left.75 \pm 2 \% \mathrm{UR}\right)$, até seu uso. Na ocasião da montagem do experimento, as sementes apresentavam $25 \%$ de umidade, determinada pelo método direto, segundo Brasil (2009).

Os tratamentos, organizados em esquema fatorial $2 \times 6$, consistiram na combinação de duas espécies (Conyza canadensis e C. bonariensis) e seis niveis de qualidade de luz (luz branca, vermelha, azul, verde, vermelho-distante e ausência de luz).

Foram utilizadas, como unidades experimentais, caixas de acrílico transparente tipo gerbox (11,0 x 11,0 x 3,5 cm), limpas com água sanitária comercial (2,5\% de cloro ativo) diluída em água a $5 \%(\mathrm{v} / \mathrm{v})$ e, em seguida, borrifadas com álcool 70\% (v/v) e secas ao ar sobre bancada, em temperatura ambiente. Os papéis mata-borrão, utilizados como substratos, foram envolvidos em folhas de alumínio flexivel e esterilizados em autoclave a $120^{\circ} \mathrm{C}$ por três horas.

Cada parcela foi constituída de uma caixa gerbox contendo 50 sementes. As sementes foram distribuídas uniformemente dentro das caixas gerbox, dispondo-as sobre duas folhas de papel mata-borrão secas. Após a distribuição das sementes em todos os tratamentos, foi realizado o umedecimento com água destilada, seguido do imediato revestimento das caixas com papel-celofane, para obtenção das diferentes cores de luz. Todas as parcelas foram mantidas dentro de câmaras de germinação reguladas para $25^{\circ} \mathrm{C}$ e 12 horas de luz.

Para obtenção das diferentes qualidades de luz, as caixas de germinação foram envolvidas por duas folhas de papel-celofane de cor correspondente ao tratamento; para o tratamento vermelho-distante, elas foram envolvidas por duas folhas de papel-celofane vermelho e duas de azul. Não se usou revestimento para o tratamento com luz branca (testemunha) (Toledo et al., 1993; Lopes et al., 2005; Yamashita et al., 2008).

Para verificar a passagem da luz pelos diferentes materiais utilizados, foram realizadas leituras de porcentagem da transmitância em um espectrofotômetro UV-Visivel da marca $\operatorname{Varian}^{\circledR}$, modelo Cary 50 Conc. Como célula de leitura, para disposição das amostras, foi utilizada uma cubeta de quartzo com caminho óptico de $10 \mathrm{~mm}$, onde os papéis foram colocados em folhas duplas, conforme utilizados sobre as caixas gerbox, realizando-se as leituras em diferentes comprimentos de onda (Tabela 1).

A germinabilidade (protrusão de raiz primária) foi avaliada aos cinco e 10 dias do início do experimento. Tanto a montagem quanto a verificação da germinação foram realizadas em câmara equipada com luz verde, cujo comprimento de onda, na faixa de 610 e $650 \mathrm{~nm}$, tem sido considerado como seguro nas avaliações de germinação em tratamentos de escuro (Cardoso, 1995).

Após a segunda contagem (10 dias), as sementes remanescentes de todos os tratamentos receberam luz branca, sendo avaliado o número de sementes germinadas diariamente durante mais 10 dias. Concluído esse período, foi verificada a vitalidade das sementes não germinadas, por meio do teste da "pressão ao toque", seguindo metodologia

Tabela 1 - Valores de transmitância (\%), em diferentes comprimentos de onda, obtidos nos papéis utilizados como filtros de luz

\begin{tabular}{|c|r|c|c|c|}
\hline \multirow{2}{*}{$\begin{array}{c}\text { Comprimento } \\
\text { de onda } \\
(\mathrm{nm})\end{array}$} & \multicolumn{4}{|c|}{ Cor } \\
\cline { 2 - 5 } & Azul & Vermelho & Verde & $\begin{array}{c}\text { Vermelho } \\
+ \text { azul }\end{array}$ \\
\hline 340 & 30,07 & 26,03 & 43,04 & 8,02 \\
\hline 360 & 31,83 & 19,19 & 33,00 & 5,59 \\
\hline 380 & 35,63 & 20,58 & 28,21 & 9,08 \\
\hline 400 & 47,08 & 24,98 & 31,88 & 11,42 \\
\hline 630 & 2,32 & 64,99 & 13,61 & 1,51 \\
\hline 645 & 3,53 & 68,86 & 18,73 & 2,66 \\
\hline 650 & 4,95 & 71,73 & 22,31 & 3,53 \\
\hline 660 & 8,47 & 67,37 & 36,52 & 5,84 \\
\hline 665 & 11,41 & 69,33 & 44,07 & 8,18 \\
\hline 666 & 12,18 & 68,96 & 45,49 & 8,32 \\
\hline 670 & 15,20 & 72,83 & 51,69 & 10,48 \\
\hline 675 & 18,24 & 71,15 & 58,54 & 13,59 \\
\hline 700 & 41,75 & 71,12 & 74,64 & 28,91 \\
\hline 730 & 62,28 & 74,02 & 78,51 & 41,89 \\
\hline 750 & 68,03 & 71,26 & 78,30 & 49,75 \\
\hline
\end{tabular}


descrita por Isaac \& Guimarães (2008). Foram consideradas dormentes as sementes embebidas e não germinadas que, ao final do teste (20 dias), mantiveram aspecto firme (sementes firmes) ao serem pressionadas manualmente por estilete.

Com os dados de germinação, foi calculada a germinabilidade aos 5 e 10 dias e a germinação acumulada.

Foi utilizado o delineamento inteiramente casualizado, com quatro repetições para todos os tratamentos. Os dados foram submetidos à análise de variância (ANOVA) de cada variável dependente, verificando-se a significância dos fatores principais e de suas interações, com base no teste $F(\alpha=0,05)$.

\section{RESULTADOS E DISCUSSÃO}

Na contagem do número de sementes germinadas aos 5 e 10 dias, foi observada diferença entre os tratamentos de qualidade de luz $(\mathrm{p}<0,05)$. A germinabilidade final foi influenciada pela espécie e pela qualidade de $\operatorname{luz}(\mathrm{p}<0,05)$, não havendo efeito da interação entre os fatores $(p>0,05)$.

Na primeira contagem, realizada aos cinco dias, a luz branca e a vermelha proporcionaram germinabilidade acima de 90 e $70 \%$, respectivamente, enquanto nos demais filtros essa variável não ultrapassou 5\%. Esses resultados se mantiveram na avaliação aos 10 dias, quando a germinação atingiu 95,5\% para luz branca e $83,3 \%$ para vermelha (Tabela 2).

A luz atuou como fator determinante para a germinação das sementes de Conyza, e o envolvimento dos tratamentos com o celofane reduziu significativamente a sua germinação. Entretanto, sob luz branca, houve maior conversão de Fv (fitocromo na forma inativa) para Fve (forma ativa do fitocromo) e, como para maior parte das sementes fotoblásticas, como é o caso das espécies de Conyza estudadas, promoveu-se a germinação.

Apesar de a luz vermelha ter promovido germinação superior a $70 \%$ na primeira contagem e de $83 \%$ na segunda, ainda assim, essa condição não foi suficiente para ser equiparada a valores obtidos sob luz branca. Esse comportamento pode ser indicativo de que a luz vermelha de ter reduzido a intensidade luminosa que chegava às sementes, diminuindo o percentual de germinação delas.

Esses resultados são coerentes com os relatos de Bewley \& Black (1994), de que o comprimento de onda da luz que promove a germinação em maiores percentuais encontra-se entre 650 e $700 \mathrm{~nm}$ (vermelho), a esse processo é inibido a $730 \mathrm{~nm}$ (vermelhodistante). A maior parte das sementes de espécies que respondem à luz não é domesticada (Baskin \& Baskin, 1988), como é o caso das plantas daninhas, em que a resposta morfogenética, regulada pelo fitocromo, depende do comprimento de onda adequado para a germinação.

O requerimento de luz é o principal motivo pelo qual a germinação das sementes é restrita à proximidade da superfície do solo (Toledo et al., 1993). Como muitos solos atenuam efetivamente a luz, o que passa, tanto em quantidade como em qualidade, não é suficiente para induzir a germinação. As sementes das espécies estudadas são pequenas e apresentam pouca reserva nutritiva (Loux et al., 2010); assim, a luz pode ser um fator de indução à germinação, para que esta ocorra apenas sobre ou próxima à superficie do solo.

Em solos agrícolas, muitas sementes são revolvidas pelas práticas de preparo e cultivo do solo, e, com essa característica, elas germinam apenas quando reexpostas à luz no cultivo subsequente (Wooley \& Stoller, 1978; Toledo et al., 1993). As sementes de plantas daninhas, como é o caso de Conyza, também podem detectar a presença de concorrentes potenciais (cobertura vegetal espessa, sombreamento de plantas vizinhas), pela ausência de luz no comprimento de onda adequado, reduzindo assim a probabilidade de competição e aumentando a de sobrevivência.

Tanto para Conyza canadensis como para C. bonariensis, os comprimentos de onda proporcionados pela luz verde, azul e vermelhodistante reduziram a germinabilidade das sementes, com média inferior a 5\%. A absorção diferencial dos comprimentos de onda do espectro da luz que chegaram até as sementes foi insuficiente para desencadear o processo germinativo. 
Tabela 2 - Germinação após 5 e 10 dias da montagem do experimento (\%) e germinação acumulada (\%) de sementes de Conyza canadensis e C. bonariensis mantidas sob diferentes qualidades de luz. Alta Floresta-MT, 2010

\begin{tabular}{|c|c|c|c|}
\hline Fator & $\begin{array}{c}\text { Germinação aos } 5 \text { dias } \\
(\%)\end{array}$ & $\begin{array}{c}\text { Germinação aos } 10 \text { dias } \\
(\%)\end{array}$ & $\begin{array}{c}\text { Germinação acumulada } \\
(\%)\end{array}$ \\
\hline \multicolumn{4}{|l|}{ Espécie (E) } \\
\hline Conyza canadensis & $26,7 \mathrm{a}$ & $29,7 \mathrm{a}$ & $88,2 \mathrm{~b}$ \\
\hline Conyza bonariensis & $29,7 \mathrm{a}$ & $31,6 \mathrm{a}$ & $95,3 \mathrm{a}$ \\
\hline Valor de F & $3,499^{\mathrm{ns}}$ & $2,799^{\mathrm{ns}}$ & $39,481^{*}$ \\
\hline \multicolumn{4}{|l|}{ Qualidade de luz (Q) } \\
\hline Azul & $0,0 \mathrm{c}$ & $0,0 \mathrm{c}$ & $91,0 \mathrm{~b}$ \\
\hline Verde & $3,5 \mathrm{c}$ & $4,3 \mathrm{c}$ & $90,3 \mathrm{~b}$ \\
\hline Vermelho & $71,8 \mathrm{~b}$ & $83,3 \mathrm{~b}$ & $92,8 \mathrm{ab}$ \\
\hline Vermelho-distante & $0,3 \mathrm{c}$ & $0,5 \mathrm{c}$ & $89,0 \mathrm{~b}$ \\
\hline Branca & $91,3 \mathrm{a}$ & $95,5 \mathrm{a}$ & $97,0 \mathrm{a}$ \\
\hline Ausência de luz & $0,5 \mathrm{c}$ & $0,3 \mathrm{c}$ & $90,3 \mathrm{~b}$ \\
\hline Valor de F & $705,028 *$ & $1.060,759^{*}$ & $4,320 *$ \\
\hline \multicolumn{4}{|l|}{ Interação (E x Q) } \\
\hline Valor de F & $0,753^{\mathrm{ns}}$ & $0,704^{\mathrm{ns}}$ & $1,776^{\mathrm{ns}}$ \\
\hline $\mathrm{CV}(\%)$ & 16,05 & 12,96 & 4,26 \\
\hline
\end{tabular}

${ }^{\mathrm{ns}}$ não significativo pelo teste $\mathrm{F} .{ }^{*}$ significativo a $5 \%$ de probabilidade pelo teste $\mathrm{F}$.

No campo, o sombreamento por vegetação produz uma relação vermelho/vermelhodistante baixa, reduzindo ou inibindo a germinação de várias espécies, que por sua vez germinam rapidamente sob luz branca (Schmitt \& Wulff, 1993; Toledo et al., 1993; Ballaré \& Casal, 2000). As espécies de plantas daninhas respondem de forma diferencial à presença de comprimentos de onda específicos: não houve indução de germinação de Xanthium strumarium quando foram colocados sob luz branca e verde (Toledo et al., 1993); já Basella rubra e Porophyllum ruderale não sofreram influência de luz vermelha e vermelho-distante (Lopes et al., 2005; Yamashita et al., 2008, respectivamente).

As sementes de ambas as espécies de Conyza retomaram o processo germinativo após a retirada do celofane que envolvia os tratamentos, apresentando germinação superior a $90 \%$ no final do experimento. Contudo, à exceção da luz vermelha, que não diferiu da luz branca, as demais qualidades de luz proporcionaram germinação inferior a essa. A retomada do processo germinativo ocorreu entre três e cinco dias (Figura 1), sendo observada protrusão da raiz primária.
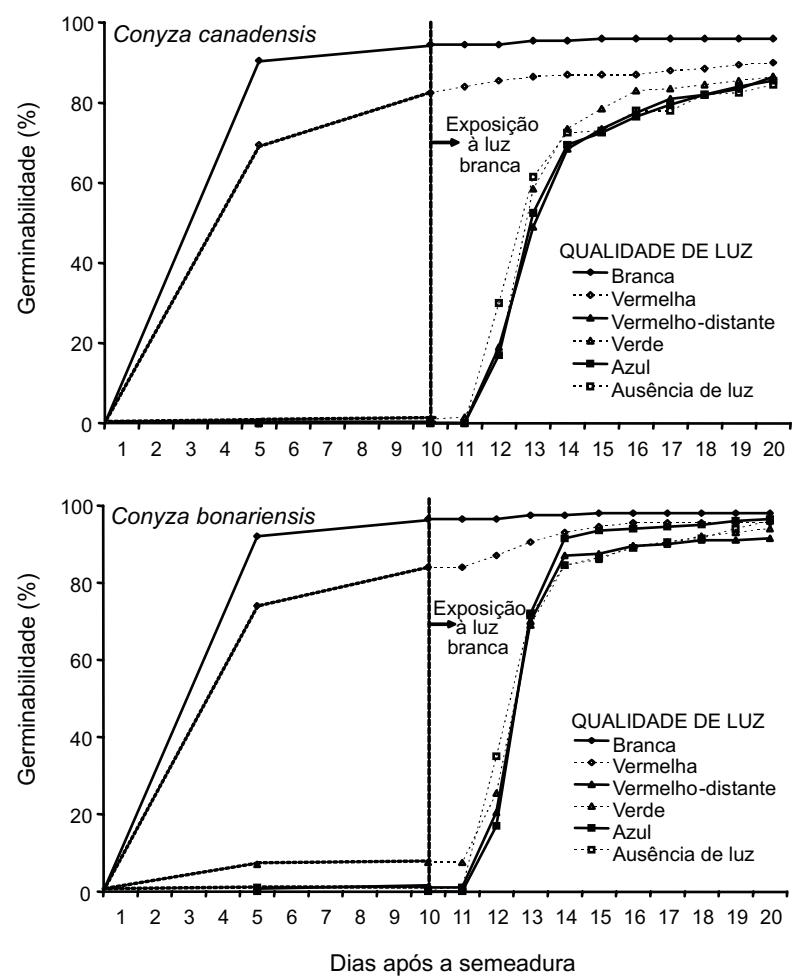

Figura 1 - Germinabilidade acumulada de sementes de Conyza canadensis e C. bonariensis sob diferentes qualidades de luz. Alta Floresta-MT, 2010

Planta Daninha, Viçosa-MG, v. 29, n. 4, p. 737-743, 2011 


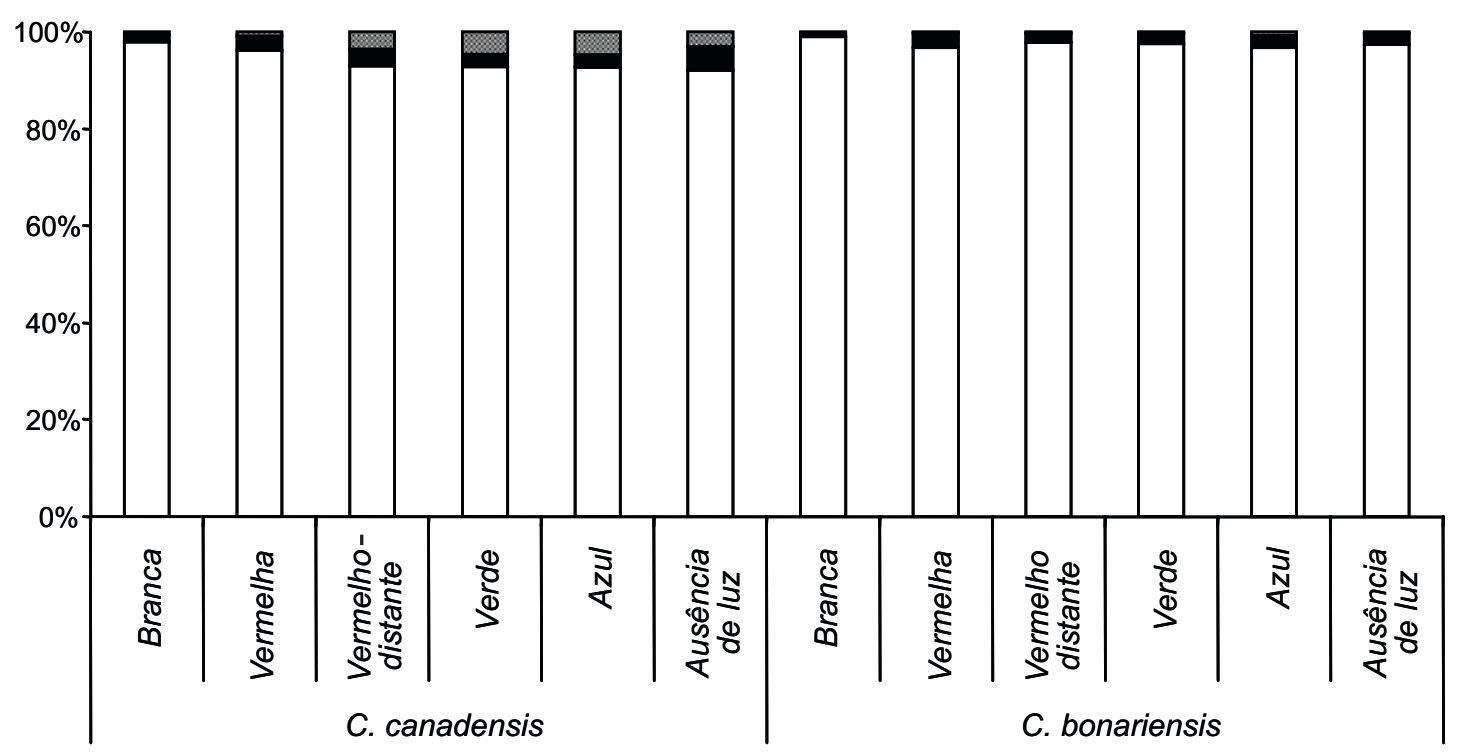

Germinadas

Mortas

Firmes

Figura 2 - Porcentagem de sementes de Conyza canadensis e C. bonariensis germinadas, mortas e firmes, ao final do experimento de qualidade de luz. Alta Floresta-MT, 2010.

Sementes não germinadas de Conyza canadensis apresentaram maiores percentuais de sementes firmes (entre 3,5 e 9,0\%) que C. bonariensis (entre 0,5 e 4,0\%), ou seja, um maior número de sementes caracterizadas como viáveis (Figura 2). O percentual das sementes mortas, independentemente da espécie estudada, foi inferior a 4,5\%.

De acordo com os dados obtidos no presente trabalho, conclui-se que as sementes dessas duas espécies germinam apenas na presença de luz, sendo, portanto, fotoblásticas positivas. A qualidade da luz interfere na germinação das sementes nas duas espécies, ocorrendo maior germinabilidade sob luz branca, seguida da luz vermelha. Isso demonstra que, dependendo da qualidade de luz advinda do material usado como cobertura do solo, a germinação dessas espécies pode ser reduzida.

\section{LITERATURA CITADA}

BALLARÉ, C. L.; CASAL, J. J. Light signals perceived by crop and weed plants. Field Crops Res., v. 67, n. 2, p. $149-160,2000$.

BASKIN, C. C.; BASKIN, J. M. Seeds: ecology, biogeography, and evolution of dormancy and germination. San Diego: Academic Press, 1998. 666 p.

Planta Daninha, Viçosa-MG, v. 29, n. 4, p. 737-743, 2011
BEWLEY, J. D.; BLACK, M. Seeds: physiology of development and germination. 2.ed. New York: Plenum Press, 1994. $445 \mathrm{p}$.

BRASIL. Ministério da Agricultura e Reforma Agrária. Secretaria Nacional de Defesa Agropecuária. Departamento Nacional de Produção Vegetal. Coordenação de Laboratório Vegetal. Regras para análise de sementes. Brasília: 2009. $365 \mathrm{p}$.

CARDOSO, V. J. M. Germinação e fotoblastismo de sementes de Cucumis anguria: influência da qualidade da luz durante a maturação e secagem. R. Bras. Fisiol. Veg., v. 7, n. 1, p. $75-80,1995$

CASAL, J. J.; SÁNCHEZ, R. A. Phytochromes and seed germination. Seed Sci. Res., v. 8, n. 3, p. 317-329, 1998.

CHACHALIS, D.; REDDY, K. N. Factors affeting Campsis radicans seed germination and seedling emergence. Weed Sci. v. 48, n. 2 , p. $212-216,2000$

IKEDA, F. S. et al. Luz e $\mathrm{KNO}_{3}$ na germinação de sementes de Tridax procumbens sob temperatura constante e alternada. Planta Daninha, v. 26, n. 4, p. 751-756, 2008.

ISAAC, R. A.; GUIMARÃES, S. C. Banco de sementes e flora emergente de plantas daninhas. Planta Daninha, v. 26, n. 3, p. 521-530, 2008.

KISSMANN, K. G.; GROTH, D. Plantas infestantes e nocivas. 2. ed. São Paulo: BASF, 1999. Tomo II. 978 p. 
KLEIN, A.; FELIPPE, G. M. Efeito da luz na germinação de sementes de ervas invasoras. Pesq. Agropec. Bras., v. 26, n. 7, p. 955-966, 1991.

KOGER, C. H.; REDDY, K. N.; POSTON, D. H. Factors affecting seed germination, seedling emergence, and survival of texasweed (Capteronia palustris). Weed Sci., v. 52, n. 6, p. 989-995, 2004.

LOPES, J. C. et al. Influência de temperatura, substrato e luz na germinação de sementes de bertalha. R. Bras. Sementes, v. 27, n. 2 , p. $18-24,2005$.

LOUX, M. et al. Biology and management of horseweed 2004. Disponível em <http://www.ipm.uiuc.edu/pubs/ horseweed.pdf $>$. Acesso em: 28 mar. 2010

MENEZES, N. L. et al. Germinação de sementes de Salvia splendens Sellow em diferentes temperaturas e qualidades de luz. R. Bras. Sementes, v. 26, n. 1, p. 32-37, 2004.

MEROTTO Jr., A. et al. Interferência das plantas daninhas sobre o desenvolvimento inicial de plantas de soja e arroz através da qualidade da luz. Planta Daninha, v. 20, n. 1, p. 9-16, 2002.

NANDULA, V. K. et al. Factors affecting germination of horseweed (Conyza canadensis). Weed Sci., v. 54, n. 5, p. 898-902, 2006.
SCHMITT, J.; WULFF, R. D. Light spectral quality phytochrome and plant competition. Tree Physiol., v. 8, n. 1, p. 47-51, 1993.

TAKAKI, M. New proposal of classification of seed based on forms of phytochrome insted of photoblastism. R. Bras. Fisiol. Veg., v. 13, n. 1, p. 103-107, 2001.

THEBAUD, C.; ABBOTT, R. J. Characterization of invasive Conyza species (Asteraceae) in Europe: quantitative trait and isozyme analysis. Am. J. Bot., v. 82, n. 3, p. 360-368, 1995.

TOLEDO, R. E. B.; KUVA, M. A.; ALVES, P. L. C. A Fatores que afetam a germinação e a emergência de Xanthium strumarium L.: dormência, qualidade de luz e profundidade de semeadura. Planta Daninha, v. 11, n. 1/2, p. 15-20, 1993.

TREMMEL, C. D.; PETERSON, K. M. Competitive subordination of a piedmont old field successional dominant by an introduced species. Am. J. Bot., v. 70, n. 8, p. $1125-1132,1983$

WOLLEY, J. L; STOLLER, E. W. Light penetration and lightinduced seed germination in soil. Plant Physiol., v.61, n.4, p.597-600, 1978.

YAMASHITA, O. M. et al. Influência da temperatura e da luz na germinação de sementes de couve-cravinho (Porophyllum ruderale (Jacq.) Cass.). R. Bras. Sementes, v. 30, n. 3, p. 202-206, 2008. 